OPEN ACCESS

Edited by:

Liquan Liu,

Western Sydney University, Australia

Reviewed by:

Caicai Zhang,

Hong Kong Polytechnic University,

Hong Kong

Zhen Qin,

Shanghai Jiao Tong University, China

${ }^{*}$ Correspondence:

Shanshan Fan

fanshanshan@blcu.edu.cn

Specialty section:

This article was submitted to

Language Sciences,

a section of the journal

Frontiers in Psychology

Received: 30 August 2017 Accepted: 26 February 2018

Published: 23 March 2018

Citation:

Fan S, Li A and Chen A (2018) Perception of Lexical Neutral Tone

Among Adults and Infants.

Front. Psychol. 9:322.

doi: 10.3389/fpsyg.2018.00322

\section{Perception of Lexical Neutral Tone Among Adults and Infants}

\author{
Shanshan Fan ${ }^{1,2 *}$, Aijun $\mathrm{Li}^{2}$ and Ao Chen ${ }^{3,4}$ \\ ${ }^{1}$ School of Preparatory Education, Beijing Language and Culture University, Beijing, China, ${ }^{2}$ Institute of Linguistics, Chinese \\ Academy of Social Sciences, Beijing, China, ${ }^{3}$ School of Communication Science, Beijing Language and Culture University, \\ Beijing, China, ${ }^{4}$ Utrecht Institute of Linguistics OTS, Utrecht University, Utrecht, Netherlands
}

Neutral tone (TO) is a special tone form in Mandarin that contains tonal and stress information. Compared with canonical tones, T0 has a much shorter duration and reduced pitch contour. Its tonal contour is determined by the preceding canonical tone. However, not much is known about the perception of tonal and stress information in TO. In the current study, we investigate (1) whether TO can be perceived as lexically unstressed by stress-language listeners; and (2) how Mandarin (tone language)and Dutch (stress language)-learning infants perceive T0. Three experiments were conducted. In Experiment 1, Dutch adults identified T0 as unstressed when presented with disyllabic sequences ending in TO. In Experiment 2, we used the visual fixation paradigm to test 4- to 6-month-old and 10- to 12-month-old Dutch and Mandarin infants on pseudoword discrimination (/pan1san4/ [high-level + high-falling] and /pan1san0/ [high-level + mid-falling]). T4 and T0 each exhibit a similar falling contour. The results show that (1) after being habituated to neutral tone sequences (/pan1san0/), Dutch infants discriminated the T1T0-T1T4 contrast; and (2) neither age groups of Mandarin infants discriminated the tone contrast. Assuming Mandarin infants' lack of discrimination might be due to the similar FO contours, we tested Mandarin infants in Experiment 3 using a more salient contrast, /pan1san2/ (high-level + mid-rising) and /pan1san0/. While no overall discrimination was observed, those who were habituated to /pan1san0/ demonstrated discrimination. The continuous discrimination of Dutch infants suggests that they might process neutral-canonical tone contrast as lexical stress rather than as tonal information. Overall, Mandarin infants' failure implies that the representation of TO is not complete during their 1st year of life; the acquisition of tonal categories may therefore take longer than we expected.

Keywords: perceptual reorganization, tone acquisition, lexical neutral tone, lexical stress, cross-language comparison

\section{INTRODUCTION}

Lexical tones are pitch variations that distinguish lexical meanings. Mandarin is the most widely studied tone language, in which four canonical tones are used to distinguish word meanings, including T1 (high-level; 55 in Chao tone letters), T2 (mid-rising; 35), T3 (low-dipping; 21/214) and T4 (high-falling; 51). For example, the following words have different meanings based on canonical tones: /ma1/ (妈, mother), /ma2/ (麻, numb), /ma3/ (马, horse), and /ma4/ (骂, to scold). Besides the four canonical tones, neutral tone (T0) never occurs independently or at the beginning of a word. It is always preceded by a canonical tone. Neutral tone can distinguish word meanings, 
such as /tu 1 1 $\mathrm{ci} 1 /$ (东西, east and west) and /tu 11 ci0/ (东西, things), and appear in different lexical and syntactic contexts, including reduplication, affixation, lexeme type, directional complements, complement particles, etc. With regard to lexeme type, words are distinguished solely by the presence of neutral tone without any other morphological or grammatical marker, such as /tu116i1/ (东西, east and west) vs. /tuy1cio/ (东西, things) (Luo and Wang, 2002; Lin, 2012). In the present study, we focus on the lexeme type.

Neutral tone is acoustically light with a shorter duration and reduced pitch contour. It has been referred to as unstressed or weak stress in previous studies (Chao, 1979; Zhu, 2002; Lu and Wang, 2005; Wei, 2005; Duanmu, 2007; Cao, 2008; Deng, 2010; Jia, 2011; Bao and Lin, 2014). The tonal contour of T0 is determined by the preceding canonical tone. When preceded by $\mathrm{T} 1, \mathrm{~T} 2$, or $\mathrm{T} 4$, the tonal contour of $\mathrm{T} 0$ is falling; when preceded by T3, the tonal contour is mid-level (Chao, 1979; Wu, 1992; Kong and Lü, 1998; Luo and Wang, 2002; Lin and Wang, 2013; Zhang and Li, 2016). Neutral tone has a lower pitch register and narrower pitch range. Pitch patterns are shown in Figure 1, where the dashed lines denote sequences ending with a neutral tone. The duration of neutral tone is about $50 \%$ of its corresponding canonical tone (Lin and Yan, 1980; Lin, 1983; Lee, 2003) or about $60 \%$ of the preceding canonical tone (Cao, 1986; Li, 2017). In summary, neutral tone contrasts with canonical tone lexically because the neutral tone is unstressed and has distinguished pitch pattern. Neutral tone possesses properties of lexical stress and lexical tone.

The acoustic correlates of neutral tone are duration, F0, intensity, and spectral features (i.e., vowel reduction, initial consonant voicing, and spectral tilt steeping). The main acoustic correlates of neutral tone are F0 and duration (Lin and Yan, 1980; Lin, 1983; Cao, 1986; Yang, 1989; Wang, 2004; Chen and Xu, 2006; Li and Fan, 2015), with F0 being more important than duration (Cao, 1986; Wang, 2004; Li and Fan, 2015; Li, 2017). Spectral tilt is a reliable cue, but it is less important than duration (Zhong et al., 2001). Intensity is not reliable (Lin and Yan, 1980; Lin, 1983). The same acoustic correlates are found for lexical stress in stress language, with duration being the most reliable cue for lexical stress in Dutch (Sluijter and van Heuven, 1995, 1996; van Heuven and de Jonge, 2011).

Previous research has revealed inconsistencies regarding how infants perceive lexical tones and lexical stress early in life. Some studies found supportive evidence for the perceptual reorganization of lexical tones, which occurred around 9 months. For example, prior to 6 months, both tone- and non-tonelanguage infants can discriminate lexical tones. By around 9 months, non-tone-language infants' sensitivity to lexical tones declines, whereas no such decline is observed among tonelanguage infants (Mattock and Burnham, 2006; Mattock et al., 2008). Some other studies, however, reported different results. For instance, in Liu and Kager (2014), 5- to 18-month-old Dutch infants showed continuous discrimination of Mandarin T1-T4 contrast. But when the phonetic distance between $\mathrm{T} 1$ and $\mathrm{T} 4$ was reduced, the infants no longer demonstrated discrimination. In Chen and Kager (2016), 4-month-old Dutch infants failed to discriminate a non-salient Mandarin tonal contrast (T2-T3), yet 6- and 12-month-old infants succeeded. Infants may not be born with the ability to discriminate all the native contrasts and may especially need time to learn phonetically non-salient contrasts (Sundara et al., 2006; Narayan et al., 2010). For lexical tones, Shi (2010) discovered that Mandarin infants were only able to categorize phonetically variable lexical tones gradually after 8 months. In Tsao (2008), 12-month-old Mandarin infants discriminated $\mathrm{T} 1-\mathrm{T} 3$ better than $\mathrm{T} 2-\mathrm{T} 3 / \mathrm{T} 2-\mathrm{T} 4$ contrasts. Taken together, early discrimination of lexical tones appears to exhibit a complex developmental pattern, where successful discrimination might relate to the phonetic salience of particular tonal contrasts.

In terms of lexical stress, in studies supporting perceptual reorganization, infants' stress perception appears to shift from universal discrimination to their native language at 9 months of age (Sansavini et al., 1997; van Ooijen et al., 1997; Hohle et al., 2009; Skoruppa et al., 2009, 2013). For example, newborn French infants could discriminate stress-initial and stress-final words (Sansavini et al., 1997), while 9-month-old French infants failed to discriminate stress contrast at a phonological level. Hence, French infants adapted their stress perception to their native language by 9 months. Nine-month-old Spanish infants, whose native language has contrastive lexical stress, demonstrated discrimination (Skoruppa et al., 2009). In some other studies, however, the discrimination of contrastive lexical stress requires sufficient exposure to ambient input (Weber et al., 2004; Keij and Kager, 2013; Butler et al., 2015). For instance, 5-monthold German infants could discriminate between stress-initial and stress-final pseudowords, yet 4-month-old German infants could not (Weber et al., 2004). In summary, attunement seems flexible in early language perception. It might be modulated by ambient language input for lexical tone and lexical stress. For lexical tone, participants' discrimination could be related to the acoustic salience of particular stimuli.

Besides acoustic salience, the order of stimuli presentation may influence the discrimination effect as well. Perceptual asymmetry was found in previous studies on the discrimination of both segments (Polka and Werker, 1994; Polka and Bohn, 1996, 2003) and suprasegments (Weber et al., 2004, 2005; Tsao, 2008; Chen, 2013; Segal et al., 2016). In Segal et al. (2016), when discriminating between initial and final lexical stress, Hebrew infants showed better discrimination when presented with uncommonly initial stress first. German-learning infants also showed similar perceptual asymmetry when perceiving lexical stress, namely that change detection was easier for infants when trochee, the predominant stress pattern, was embedded in iambs rather than the other way around (Weber et al., 2004). For early perception of lexical tones, Mandarin infants discriminated the T1-T3 contrast better if they were presented with $\mathrm{T} 1$ first than the other way around (Tsao, 2008). The mechanism underlying such asymmetry is not fully understood, yet it may be related to statistical distribution in the input. When habituated to an atypical pattern in ambient input, infants may consolidate such a pattern in representation and subsequently discriminate the frequent pattern in the input from the infrequent one. Yet if infants are habituated to the frequent pattern in the input, they might perceive the infrequent pattern as a non-prototypical realization of the frequent one. 

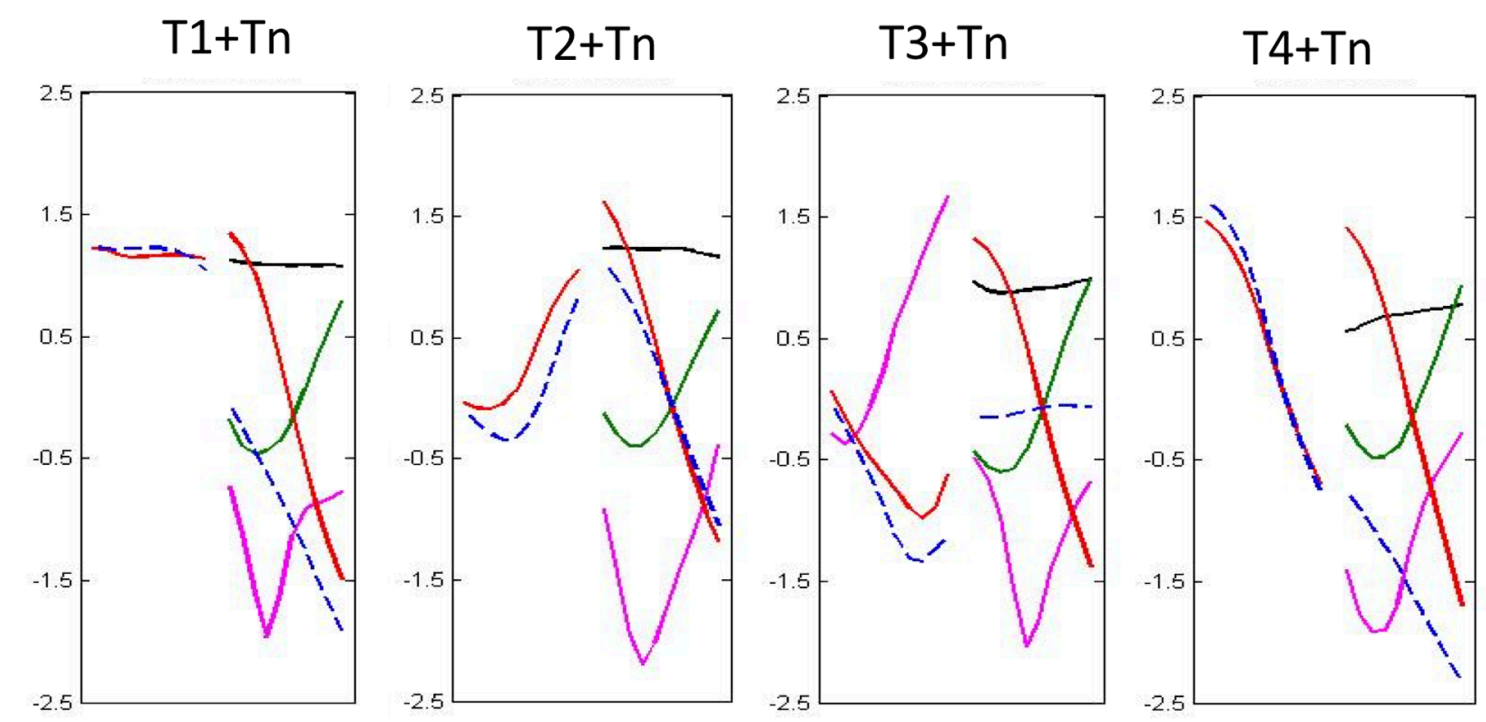

FIGURE 1 | FO contour patterns of all possible disyllabic tonal combinations (dashed lines represent neutral tone combinations). The vertical axis is the normalized $z$-score; the horizontal axis is the normalized duration (Li and Gao, 2017).

The statistical distribution of particular phonological features in the input influences infants' perceptions of such features. Scholars have largely agreed that infants are sensitive to statistical distribution in speech input (e.g., Saffran et al., 1996; Maye et al., 2002). Infants prefer predominant patterns to which they are exposed in their native language, and such preferences are established with accumulating exposure (Jusczyk et al., 1993). In the current study, we compared stress-language (Dutch) and tone-language (Mandarin) infants on their discrimination of canonical and neutral tones. Because neutral tone carries lexical stress and tonal information, it serves as a feasible means to investigate early attunement to lexical tone or stress as the result of ambient input. We posed the following questions in the current study: (1) whether Mandarin infants can discriminate between neutral and canonical tones, and whether such discrimination is influenced by acoustical salience of the tones; and (2) whether Dutch listeners perceive neutral tone as tonal or as lexical stress, and whether perceptual reorganization can be observed for neutral tone. We began by testing whether tone- and stresslanguage-speaking adults perceived neutral tone as unstressed, which served as a baseline for the subsequent infant experiments. Next, we tested 4- to 6-month-old and 10- to 12-month-old Dutch and Mandarin infants on their discrimination of Mandarin canonical-neutral tone contrast. If Dutch infants perceived the canonical-neutral tone contrast as lexical stress, we would expect successful discrimination at both ages; on the other hand, if they perceived them as tonal, discrimination may only be successful for the younger group. For Mandarin infants, we expected them to be capable of discriminating the contrasts at both ages. Considering that sequences with neutral tone occur less frequently than those involving canonical tones, it may take time for Mandarin infants to learn these contrasts. In this case, we would expect only the 10- to 12-month-old Mandarin infants to discriminate the contrasts.

\section{EXPERIMENT 1: ADULTS' PERCEPTIONS OF NEUTRAL TONE}

To understand whether Dutch adult listeners perceive neutral tone as unstressed, a discrimination task and an identification task were conducted in Experiment 1. In the discrimination task, participants were required to discriminate disyllabic sequences ending in a neutral tone from those ending in a canonical tone. If Dutch adult listeners perceived neutral tone as unstressed, they would discriminate canonical-neutral tone contrast successfully. In the identification task, participants were required to identify the position of stress in the disyllabic sequences. Because duration is the most reliable cue for lexical stress in Dutch, and neutral tone exhibits a shorter duration compared with canonical tones, we predicted that Dutch adult listeners would identify the neutral tone as unstressed. For Mandarin listeners, given T0 as a category in native phonology, we assumed they would succeed in the discrimination task and thus be able to identify the neutral tone as unstressed.

\section{The Discrimination Task}

\section{Stimuli}

The pseudoword /pansan/ was selected as the tone-bearing sequence, which is a well-formed sequence phonotactically in Mandarin and Dutch. All possible tone combinations were included except T3T3, which is always produced as T2T3 due to the Mandarin tone sandhi process. In total, 19 target pseudowords were obtained, including 15 disyllabics ending with a canonical tone $(4 \times 4-1=15)$ and 4 disyllabics ending with a neutral tone (TnT0; $n=1,2,3$, or 4). Another 20 tonal pairs of real words in Mandarin were added as fillers, which carried the same segments but different canonical tones, such as /ş $22 \mathrm{tsg}^{\mathrm{h}} \mathrm{a} \mathrm{\eta}_{2} /$ (时长, duration) vs. /ş $44 \mathrm{ts}^{\mathrm{h}} \mathrm{a} \mathrm{y} 3 /$ (市场, market). 
All stimuli were produced by a 35-year-old male native Mandarin speaker. The speaker was born and raised in Beijing. No disorder was reported related to reading, speaking, or listening. Nineteen pseudowords were recorded along with 40 filler words in the soundproof room of the phonetics lab at the Chinese Academy of Social Sciences (CASS) using Cool Edit Pro 2.0 at a sample rate of $44,100 \mathrm{~Hz}$.

\section{Participants}

Eighteen Mandarin adult listeners were tested, 10 males and 8 females, with an average age of 20.8 years $(S D=1.9)$. Another participant took part in the test but was excluded due to equipment failure. All participants were born and raised in Beijing, without reported hearing or speech disorders.

Eighteen Dutch adult listeners were tested, 6 males and 12 females, with an average age of 23.7 years $(S D=4.7)$. They were born and raised in the Netherlands. None of the participants had been exposed to any tone language, and no hearing or speech disorders were reported.

\section{Procedures}

The AX paradigm was adopted. Participants were presented with pairs of stimuli and required to indicate whether the two stimuli were the same or different. The series consisted of 30 pairs of different stimuli (AX or XA) and 19 pairs of identical stimuli (AA or XX). For each different pair, the comparison was only conducted between a sequence ending in a canonical tone and its corresponding neutral tone form. Taking /pan1san1/ as an example, its neutral tone form was "/pan $1 \operatorname{san} 0 /$ ". The different pairs were "/pan 1san0/vs. /pan1san1/" and "/pan1san1/ vs. /pan $1 \operatorname{san} 0 /$ ", and the identical pairs were "/pan $1 \operatorname{san} 1 /$ vs. $/$ pan $1 \operatorname{san} 1 /$ " and "/pan $1 \operatorname{san} 0 /$ vs. $/$ pan $1 \operatorname{san} 0 /$ ". Another 80 pairs of fillers included different pairs such as "/s $22 \operatorname{ts}{ }^{h} a \eta_{2} /$ (时长, duration) vs. /s $44 \mathrm{tsg}^{\mathrm{h}} \mathrm{a} \mathrm{\eta}_{3 /}$ (市场, market)” and identical

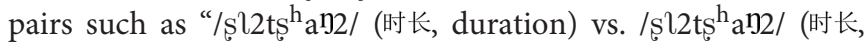
duration)."

A practice phase preceded the experiment. Seven pairs of stimuli were used to familiarize participants with the procedure. Each trial started with a fixation cross, followed by two audio stimuli with an inter-stimulus interval of $200 \mathrm{~ms}$. When the audio stimuli concluded, two buttons were shown on the screen, labeled as "Same (F)" and "Different (J)." Participants provided their response by pressing either "F (Same)" or "J (Different)" on the keyboard. The next trial started automatically after the participant had responded. The inter-trial interval was $500 \mathrm{~ms}$. ZEP was used to control the procedures, randomize stimuli, and collect participants' responses (Veenker, 2013).

\section{Results}

The accuracy rate was calculated by dividing the number of correct responses by the number of total trials for each participant. For identical pairs, the accuracy rate for Mandarin listeners was $93 \%(S D=1.24)$ and $97.1 \%$ for Dutch listeners $(S D=0.55)$. When discriminating different stimuli pairs, the accuracy rate for Mandarin listeners was 91.7\% $(S D=0.74)$ and $90.5 \%(S D=0.99)$ for Dutch listeners.

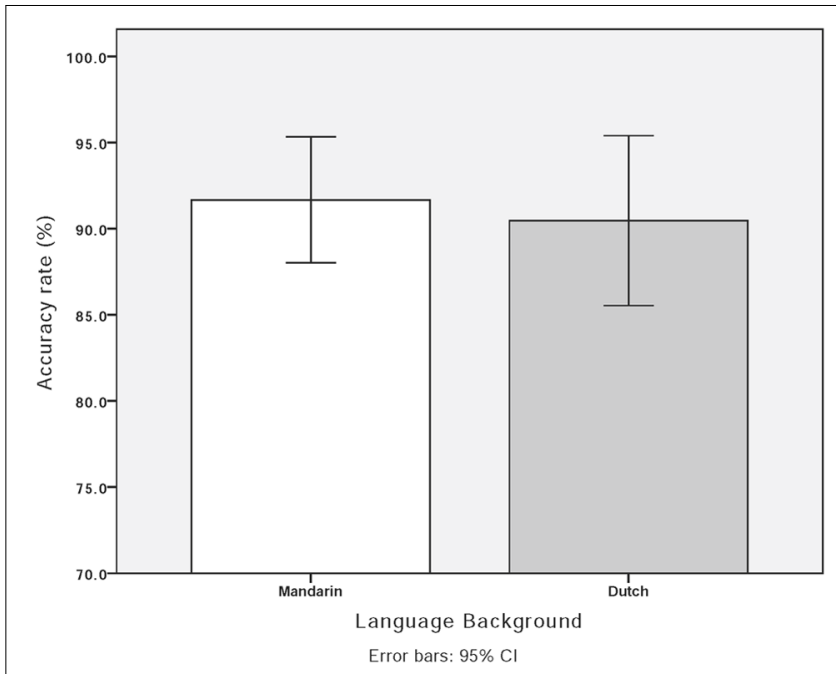

FIGURE 2 | Accuracy rate in the AX discrimination task by Mandarin and Dutch adult listeners.

Figure 2 illustrates the accuracy rates of Mandarin and Dutch adult listeners. To better understand participants' sensitivity to the canonical-neutral contrast, $d$-prime $\left(d^{\prime}\right)$ was calculated. An independent $t$-test was conducted using $d$-prime with the language group as the independent variable. No difference was found between Dutch and Mandarin adult listeners $[t(34)=-0.57, p>0.05]$.

Both Dutch and Mandarin adult listeners could discriminate neutral and canonical tones. To further investigate whether they perceived the neutral tone as unstressed, we conducted the following identification task.

\section{The Identification Task \\ Stimuli}

The stimuli in the discrimination task were used in the identification task. Participants were asked to indicate the stress position in disyllabic sequences ending in a neutral tone as well as those ending in a canonical tone.

To ensure that the Dutch participants understood the task, we used Dutch lexical stress minimal pairs as practice stimuli. We selected three minimal pairs in Dutch: "kaNON - KAnon," "voorNAAM - VOORnaam," and "SERvisch - serVIES" (capital letters denote the stressed syllables) (Cutler and van Donselaar, 2001). A female Dutch native speaker produced all minimal pairs by reading each pair twice. All recordings were completed in the phonetics lab at Utrecht University using Audacity at a sample rate of $32,000 \mathrm{~Hz}$. Five native Dutch-speaking phoneticians selected the most naturally produced pairs for the identification task.

\section{Participants}

Another 15 Mandarin listeners different from those in the discrimination task were tested, 5 males and 10 females (mean age $=21.5$ years; $S D=0.58$ ). All Mandarin listeners were born and raised in Beijing, and no hearing disorders were 
reported. Another two participants were tested but excluded due to dyslexia $(N=1)$ and a reported background in phonetics $(N=1)$.

Another 14 Dutch listeners were tested, 6 males and 8 females (mean age $=25.9$ years; $S D=8.4$ ). All participants were born and raised in the Netherlands and reported no exposure to tone languages. No hearing disorders were reported. Another 6 Dutch listeners were tested but excluded for failing to identify the lexical stress for Dutch stress minimal pairs.

\section{Procedures}

A forced-choice procedure was adopted. The experiment was preceded by a practice phase in which 12 trials were used to familiarize the participants with the task. In each trial, Dutch listeners heard one word of the Dutch stress minimal pairs, such as "kaNON." The participants were required to identify the position of lexical stress. They were asked to give their response by clicking one of the buttons labeled "Initial (Strong-weak, Sw)", "Equal”, or "Final (weak-Strong, wS)". Each word of the Dutch stress minimal pairs was repeated twice. Mandarin listeners heard 12 trials. They were presented with Mandarin disyllabic sequences, such as “/s $44 \mathrm{tsch}_{\mathrm{h}} \mathrm{a} 3 /$ (市场, market)," and were required to indicate whether the word had initial, equal, or final stress by clicking the corresponding button.

Each trial in the test phase began with a fixation cross, after which an audio stimulus was presented. Participants were required to give their responses by clicking one of the buttons labeled "Initial," "Equal," or "Final." Another two buttons were below these, labeled "Repeat" and "Next." Participants were allowed to listen to the stimulus again by clicking "Repeat." By clicking the "Next" button, participants submitted their options and activated the next trial. ZEP was used to control the procedures, randomize stimuli, and collect participants' responses (Veenker, 2013).

\section{Results}

The accuracy rate in the practice phase was calculated for Dutch listeners. Only data from participants with accuracy rates over $80 \%$ in the practice phase were submitted for further analysis, including 6 males and 8 females (mean age $=25.9$ years; $S D=8.4)$.

For each option, we calculated selection percentages under different tonal conditions, including neutral tone, canonical tone, and each individual tonal combination. Taking "Initial" as an example, the percentage was calculated by dividing the number of responses indicating "Initial" by the total number of responses. A Chi-square test was conducted to access whether participants' responses depended on their language background when presented with disyllabic sequences ending in a neutral tone. The results were not significant, $\chi^{2}(2)=0.63, p>0.05$. Hence, when presented with disyllabic sequences ending in a neutral tone, no difference appeared between Mandarin and Dutch listeners. Both groups predominantly selected "Initial" for sequences ending in a neutral tone. However, for sequences with two canonical tones, a significant relationship emerged between participants' responses and language background $\left[\chi^{2}(2)=24.15\right.$, $p<0.01, \varphi=0.24]$. Mandarin listeners tended to identify stimuli as "Equal" (56.9\%), while $23.8 \%$ of Dutch listeners selected "Initial," 35.2\% chose "Equal," and 41\% chose "Final." Participants' responses across the three options are listed in Table 1.

For particular tonal combinations ending in a neutral tone, $80 \%$ of Mandarin listeners identified T1T0 as "Sw (Initial)." Percentages for other sequences involving neutral tone were T2T0 (80\%), T3T0 (86.7\%), and T4T0 (93.3\%). Seventy-eight percent of Dutch listeners identified T1T0 as "Sw." The percentages for other sequences involving neutral tone were T2T0 (64.3\%), T3T0 (78.6\%), and T4T0 (100\%). All percentages are plotted in Figure 3.

To select the tonal contrast for the subsequent infant experiment, we also analyzed participants' identification of disyllabic sequences with two canonical tones. The ending T4 was predominantly perceived as stressed. For Mandarin listeners, the percentages selecting "wS (final)" were $80 \%$ for T1T4, 33.3\% (T2T4), 73.3\% (T3T4), and 20\% (T4T4). For Dutch listeners, the percentages selecting "wS (final)" were 50\% for T1T4, 64.3\% (T2T4), 92.9\% (T3T4), and 35.7\% (T4T4) (see Figure 4). Except for tonal combinations ending with T4, Mandarin listeners tended to perceive disyllabic sequences with two canonical tones as being of "equal weight."

\section{Discussion}

Dutch (stress language) adult listeners could discriminate disyllabic sequences ending in a neutral tone from those ending in a canonical tone. In addition, Mandarin and Dutch listeners identified a neutral tone as unstressed. Overall, Mandarin listeners tended to perceive sequences with two canonical tones as having equal stress, consistent with the fact that Mandarin generally lacks word stress.

Because neutral tone contains simultaneous tonal and stress information, the following notions warrant investigation: (1) how infants process neutral tone, and whether younger infants (4- to 6-month-olds) and elder infants (10- to 12-montholds) respond differently; and (2) whether ambient language input influences infants' perceptions. Specifically, we asked the following questions in Experiment 2: Can Mandarinand Dutch-learning infants discriminate neutral tones from canonical tones? Do stress-language-learning infants perceive neutral tone as unstressed? Given that 10- to 12-monthold infants are attuned to their native language, would 4to 6-month-old infants respond differently from 10 - to 12 month-olds? To this end, we tested 4- to 6-month-old and

TABLE 1 | The selection percentage of "Initial," "Equal," and "Final" in neutral/canonical tone combinations for Dutch and Mandarin listeners.

\begin{tabular}{lcccc}
\hline Combinations & Language & Initial (\%) & Equal (\%) & Final (\%) \\
\hline Neutral tone & Mandarin & 85 & 13.3 & 1.7 \\
& Dutch & 80.4 & 16.1 & 3.6 \\
Canonical tones & Mandarin & 10.7 & 56.9 & 32.4 \\
& Dutch & 23.8 & 35.2 & 41
\end{tabular}



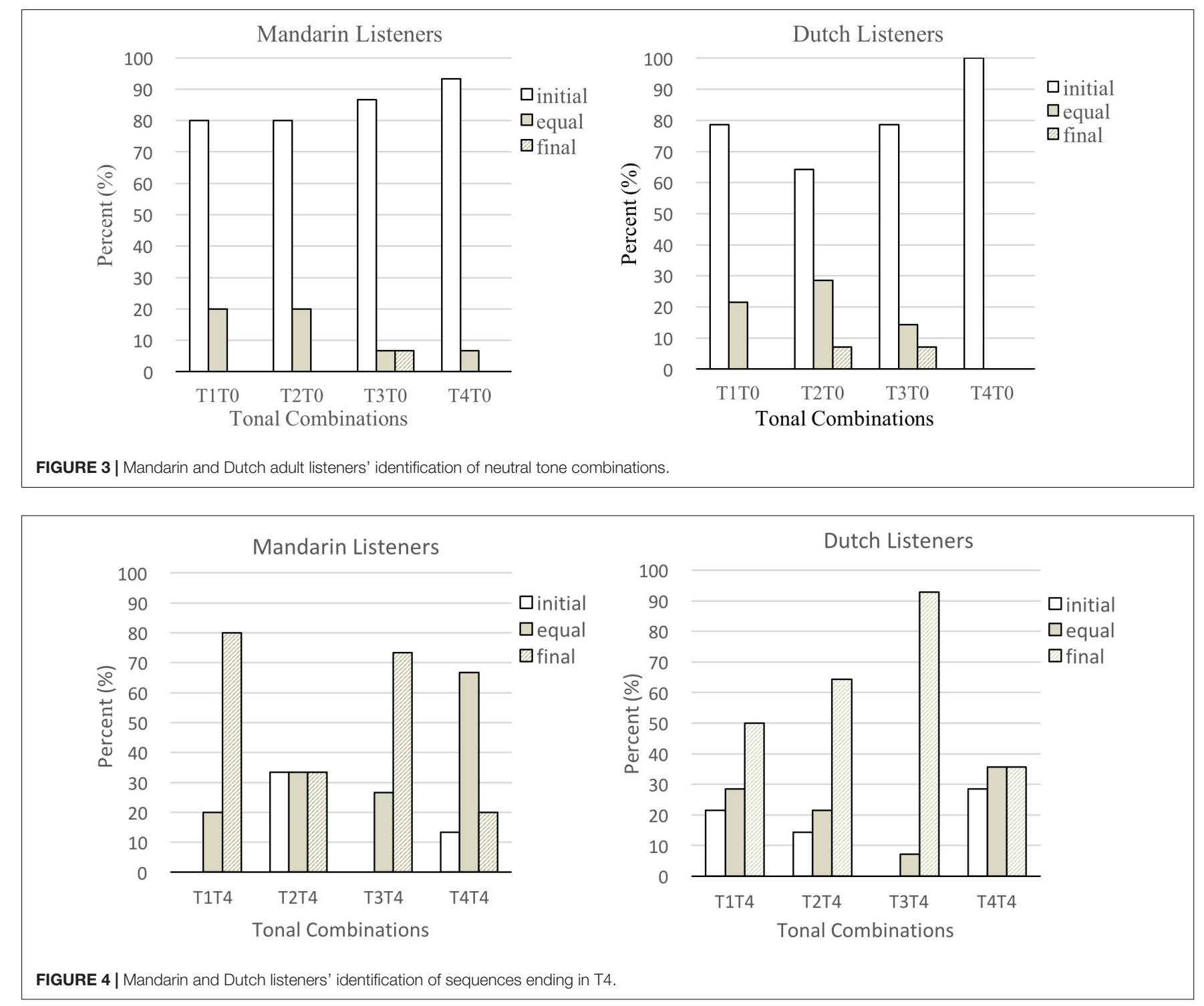

10- to 12-month-old Dutch and Mandarin infants on their discrimination of disyllabic sequences that either ended in a canonical (T4) or a neutral tone (T0). Since neutral tone is a native phonological category for Mandarin-learning infants, we predicted they would be able to discriminate between neutral tone and canonical tone throughout the 1st year of life. No difference was expected between 4- to 6-month-old and 10- to 12-month-old Mandarin infants. Given that Dutch adults were able to identify neutral tone as unstressed, if they processed neutral tone as lexical stress, they would show continuous discrimination throughout the 1 st year of life. Thus, no difference was expected between 4- to 6-month-old infants and 10- to 12-month-old Dutch infants. However, if they processed the difference between T1T4 and T1T0 as tonal information instead of lexical stress, 10- to 12-month-old Dutch infants would fail to distinguish the tonal contrast, while 4- to 6-monthold Dutch infants were expected to discriminate the contrast successfully.

\section{EXPERIMENT 2: INFANTS' DISCRIMINATION OF CANONICAL TONE AND NEUTRAL TONE}

\section{Stimuli}

The pseudoword /pansan/ was used as stimuli. In the above discrimination and identification tasks, Dutch listeners identified T1T4 as "wS (final)" and T1T0 as "Sw (initial)," respectively. Hence, we selected these two sequences as stimuli for the infant experiment.

A 32-year-old female Mandarin native speaker produced neutral tone $(/ \operatorname{pan} 1 \operatorname{san} 0 /)$ and canonical tone $(/ \operatorname{pan} 1 \operatorname{san} 4 /)$ in infant-directed speech (IDS). Each stimulus was produced 20 times. Recordings were completed in the soundproof room of the phonetics lab at CASS using Cool Edit Pro 2.0 at a sample rate of 16,000 Hz. Another five Mandarin native speakers judged the naturalness of recordings on a continuum from 1 (extremely 
unnatural) to 5 (very natural). Two phoneticians selected the six most natural tokens for T1T0 (/pan1san0/) and six most natural tokens for T1T4 (/pan1san4/).

For the canonical tone sequence/pan1san $4 /$, the average duration of the first syllable was $259.7 \mathrm{~ms}(S D=10.8)$, and the average duration of the second syllable was $316.8 \mathrm{~ms}$ $(S D=23.4)$. For the neutral tone sequence /pan $1 \mathrm{san} 0 /$, the first syllable was $269.2 \mathrm{~ms}$ on average $(S D=11.8)$, and the second syllable was $216 \mathrm{~ms}$ on average $(S D=41.8)$. For F0 contour, $10 \mathrm{~F} 0$ points were extracted along the F0 contour using Praat (Boersma and Weenink, 2013). For T1T4 combinations, the maximal F0 value of T4 was $307.4 \mathrm{~Hz}$, and its minimal F0 value was $234.3 \mathrm{~Hz}$ with a range of $136.08 \mathrm{~Hz}$. For T1T0 combinations, the maximal F0 value of T0 was $303.9 \mathrm{~Hz}$, and its minimal F0 value was $218.5 \mathrm{~Hz}$ with a range of $85.4 \mathrm{~Hz}$. The averaged F0 contours are shown in Figure 5, where T4 (high-falling) and T0 (mid-falling) exhibited similar falling contours.

\section{Participants}

Fifty-two Dutch-learning infants were tested: 23 were between 4-6 months old (mean age $=4 ; 18, S D=0.7,11$ males and 12 females), and 29 were between 10-12 months old (mean age $=11 ; 3, S D=0.8,19$ males and 10 females). Another 20 infants were tested but excluded due to fussiness $(N=6)$, parental intervention $(N=1)$, and not being habituated $(N=13)$. All Dutch-learning infants were born and raised in Dutch-speaking families where Dutch was the only language in use. All parents reported normal hearing of the infants. Dutch-learning infants were tested in the infant lab at Utrecht University (UU).

Among the 24 Mandarin-learning infants tested, 8 were between $4-6$ months old (mean age $=5 ; 9, S D=0.9,4$ males and 4 females), and 16 were between 10-12 months old (mean age $=11 ; 21, S D=1.1,6$ males and 10 females $)$. Another 16 infants were tested but excluded due to fussiness $(N=5)$, parental intervention $(N=1)$, not being habituated $(N=5)$, equipment failure $(N=3)$, dialect interference in the input $(N=1)$, and being a preterm infant $(N=1)$. All Mandarin-learning infants were born and raised in Mandarin-speaking families where Mandarin was the only language in use. All parents reported normal hearing of the infants. Mandarin-learning infants were tested in the infant lab at CASS, Beijing.

\section{Procedures}

A visual fixation procedure was adopted. During the experiment, a parent sat in a chair in the test cabin listening to music played through headphones to prevent possible intervention. The infant sat on his/her parent's lap, facing the screen in the front of the test cabin. The screen was one meter away from the infant, and the visual stimuli was played on the screen during the experiment. Two loudspeakers were situated on both sides of the cabin along with a hidden video camera above the screen. The camera was connected to a screen on the control desk, which was used to observe infants' responses to stimuli in real time. The control desk was in a separate room next to the test cabin.

There were four phases: pre-test, habituation, test, and posttest. The pre- and post-test were used to test infants' general attention. In the habituation phase, infants were habituated to either canonical tone (T1T4, /pan1san4/) or neutral tone (T1T0, /pan 1san0/). In the test phase, canonical tone and neutral tone sequences alternated between trials. In the habituation phase, three tokens from each category were used to habituate Mandarin- and Dutch-learning infants. Chen and Kager (2016) reported that 4-month-old Dutch infants could not normalize multiple tokens of tonal contrast. Thus, only another one token was used in the test phase for Dutch infants. We used another three tokens in the test phase for Mandarin infants.

Each trial started with an attention-getter. Once the infant looked at the screen, the attention-getter faded out, and the visual stimuli and audio stimuli were played. The infant's looking time and non-looking time were recorded by the experimenter on the control computer. When the average looking time of three consecutive trials was shorter than $50 \%$ of the average looking time of the first three trials, the habituation criterion was met, and the test phase started automatically. The habituation phase had a maximum of 16 trials.

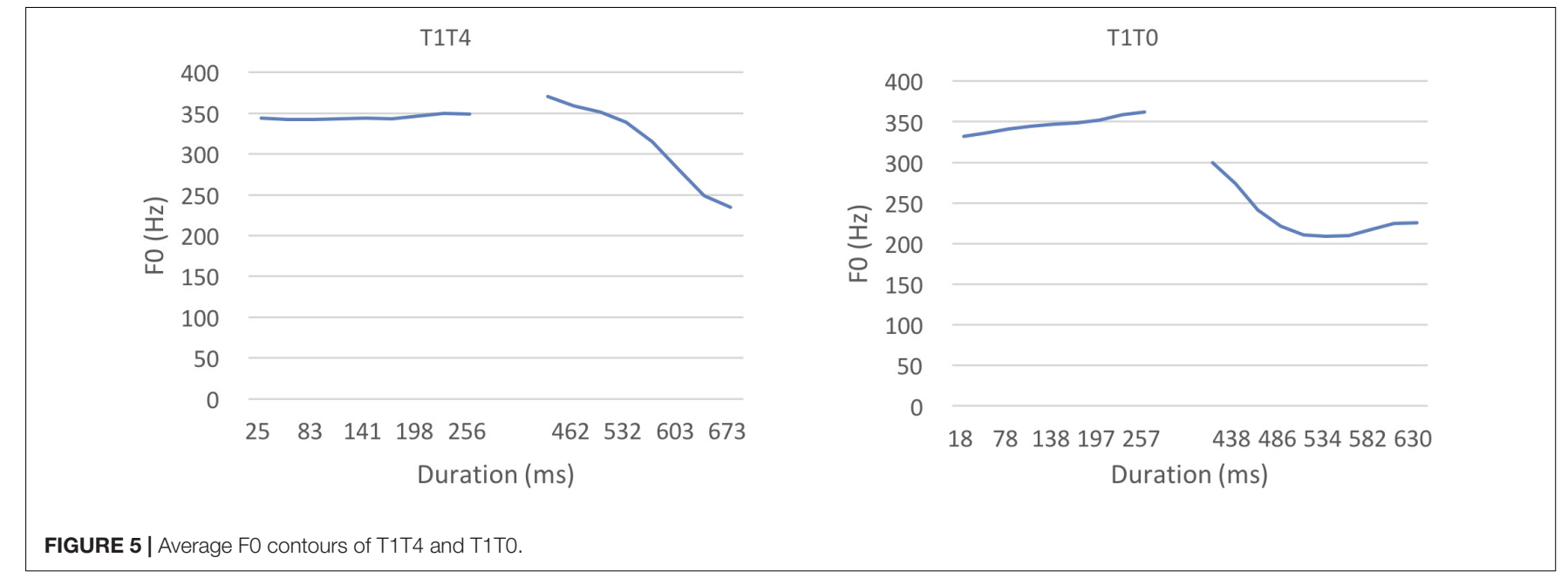


The test phase consisted of four trials, two of which were identical to the habituated tone sequences (same trials). The other two trials were the tone sequences that were not used in the habituation phase (novel trials). The same trials and novel trials alternated. Infants' looking time during the same trials and novel trials were recorded by the control computer. If they were able to discriminate the tonal sequences, then their looking time during the novel trials would presumably be longer than during the same trials.

\section{Results}

To correct for skewness, the raw looking time was logarithmically transformed. Dutch- and Mandarin-learning infants were divided into two age groups: 4- to 6-month-olds and 10- to 12month-olds. For each age group, the log-transformed looking time $(\operatorname{LogLT})$ of the same trials and of the novel trials were compared.

\section{Dutch-Learning Infants}

We conducted a 2 (trial type: same/novel) $\times 2$ (habituated category: neutral tone/canonical tone) $\times 2$ (age group: 4to 6-month-olds/10- to 12-month-olds) mixed effect ANOVA. Trial type was the within-subject factor. The between-subject factors were habituated category and age group. Trial type showed a main effect $\left[F(1,48)=6.3, p<0.05, \eta_{\mathrm{p}}^{2}=0.12\right]$ with the looking time in the novel trials being significantly longer than in the same trials $(p<0.05)$. Age group had no main effect $[F(1,48)=0.22, p>0.05]$, nor did the habituated category $[F(1,48)=0.39, p>0.05]$. There was significant interaction between trial type and the habituated category, $F(1,48)=4.5, p<0.05, \eta_{\mathrm{p}}^{2}=0.09$. When infants were habituated to T1T0, the looking time in the novel trials was significantly longer than in the same trials, $t(23)=-2.58, p<0.05$. However, when infants were habituated to T1T4, there was no difference between the same trials and the novel trials, $t(27)=-0.28, p>0.05$. Figure 6 plots the infants' looking times separated by habituated tone. Neither the interaction between trial type and age group $[F(1,48)=1.80, p>0.05]$ nor the three-way interaction among trial type, habituated category, and age group $[F(1,48)=0.04, p>0.05]$ was significant.

Although there was no interaction between trial type and age group, to better capture the perception pattern within each age group, we looked at the data for 4- to 6-month-old and 10- to 12-month-old infants separately. Dutch 4- to 6month-old infants looked longer at the novel trials (average $\operatorname{LogLT}=3.85, S D=0.27$ ) than the same trials (average $\operatorname{LogLT}=3.73, S D=0.28), t(22)=-2.26, p<0.05$. No difference was found between the same trials (average $\operatorname{LogLT}=3.74$, $S D=0.32$ ) and novel trials (average $\operatorname{LogLT}=3.78, S D=0.29$ ) for 10 - to 12 -month-old infants, $t(22)=-0.84, p>0.05$. These findings suggest that 4 - to 6-month-old infants might be more sensitive to neutral-canonical contrast than 10- to 12month-old infants. Figure 7 plots the infants' log-transformed looking time in the same and novel trials for each age group.
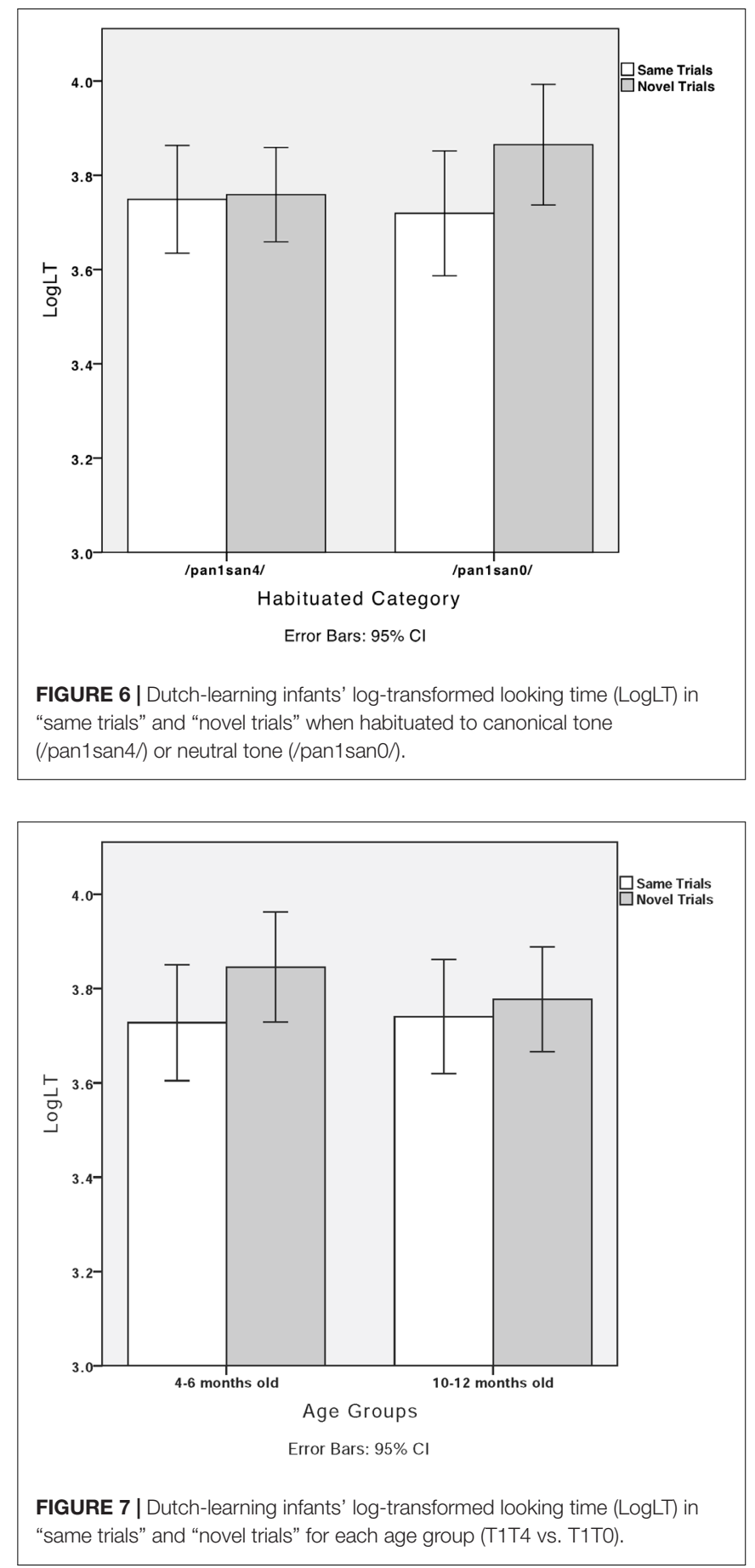

\section{Mandarin-Learning Infants}

We conducted the same 2 (trial type: same/novel) $\times 2$ (habituated category: neutral tone/canonical tone) $\times 2$ (age group: 4 - to 6month-olds/10- to 12-month-olds) mixed effect ANOVA on the data obtained from Mandarin infants. Trial type failed to show a main effect $[F(1,20)=0.03, p>0.05]$. Habituated category did not show a main effect, $F(1,20)=0.003, p>0.05$. Age group did not show significant main effect either, $F(1,20)=0.10, p>0.05$. The interaction between trial type and age group was marginally 
significant, $F(1,20)=3.58, p=0.07, \eta_{\mathrm{p}}^{2}=0.15$. No interaction was found between trial type and habituated category $[F(1,20)=0.09$, $p>0.05$ ] or among trial type, habituated category, and age group $[F(1,20)=0.31, p>0.05]$.

Because we found a marginally significant interaction between age group and trial type, we conducted paired $t$-tests with 4 - to 6-month-old and 10- to 12-month-old infants separately. The results showed no difference between the same trials and novel trials for the 4- to 6-month-old infants $[t(7)=1.41, p>0.05]$ or the 10 - to 12 -month-old infants $[t(15)=-1.61, p>0.05]$. Figure 8 shows the looking time in the same and novel trials for 4- to 6-month-old and 10- to 12-month-old infants.

\section{Discussion}

In the present experiment, we tested Dutch and Mandarin infants' discrimination of canonical (T1T4, /pan1san4/) and neutral tone (T1T0, /pan1san0/). Regardless of age, Dutch-learning infants were able to discriminate T1T4-T1T0 contrast. Given that Dutch adults perceived T1T4 and T1T0 as "wS" and "Sw," respectively, Dutch infants likely processed neutral-canonical tone contrast as lexical stress instead of tonal information. Perceptual asymmetry was found for Dutch infants: those who were habituated to T1T0 discriminated T1T4-T1T0 contrast; those habituated to T1T4 did not. For languages with a predominant initial stress pattern, such as English, German, and Dutch, infants demonstrated an initial stress preference (Fikkert, 1993; Jusczyk et al., 1993; Friederici et al., 2007). For Dutch infants, the trochaic pattern, which is T1T0 in the present study, may be more salient to perceive than the less frequent iambic T1T4 pattern. When presented with T1T0 first, it might be easier for infants to consolidate the representation of the salient pattern, which allows for later successful discrimination. When presented with the iambic

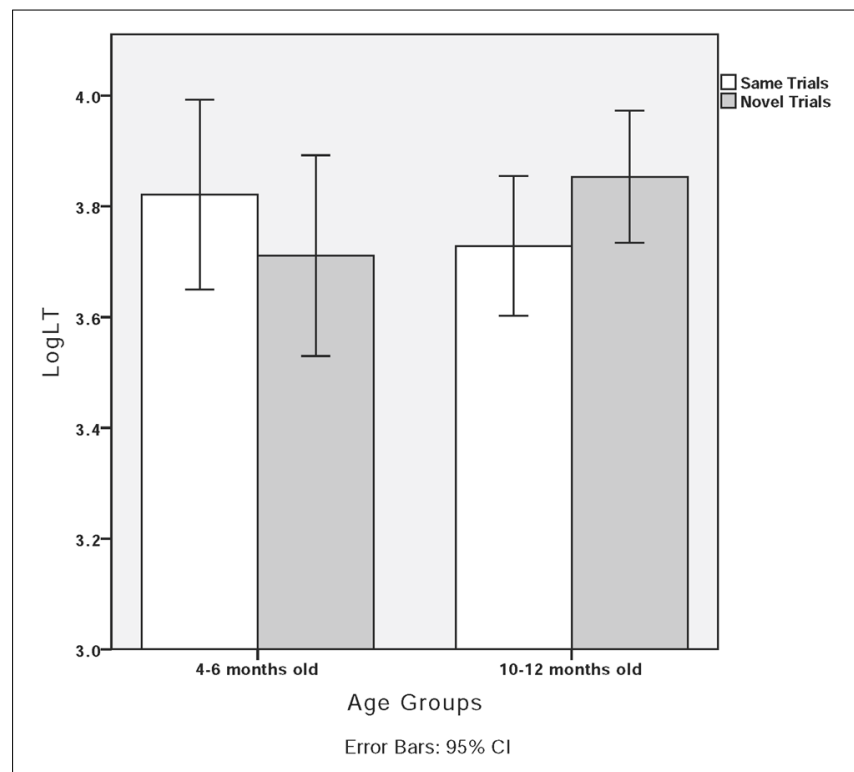

FIGURE 8 | Mandarin-learning infants' log-transformed looking time (LogLT) in "same trials" and "novel trials" for each age group (T1T4 vs. T1T0). pattern, infants may accept such a pattern as a non-prototypical realization of the trochaic pattern.

Both the 4- to 6-month-old and the 10- to 12-month-old Mandarin infants unexpectedly failed to discriminate between the neutral-canonical tone contrast, and no perceptual asymmetry emerged. In previous studies, acoustic salience influenced participants' discrimination (Tsao, 2008; Liu and Kager, 2014; Chen and Kager, 2016). With regard to T1T4 and T1T0, T4 and T0 showed a similar falling pitch contour and similar register (Li, 2017). It is possible that T0 and T4 were not distinctive enough for Mandarin infants to discriminate them. According to the assumption of perceptual assimilation model (Best, 1994), Mandarin infants might perceive T1T0 as a realization of T1T4 and vice versa. As such, the failed discrimination could have been caused by the acoustic similarities between T1T4 and T1T0.

Therefore, we tested Mandarin infants in Experiment 3 using a more salient canonical-neutral tone contrast, the T1T2-T1T0 contrast. Unlike the T1T4 and T1T0 contrast, where both T4 and T0 exhibit a falling contour, in the T1T2 and T1T0 contrast, T2 exhibits a rising contour and T0 exhibits a falling contour (see Figure 9). Compared with T4, the pitch contour of T2 is more different from T0 (Li, 2017). If the phonetic similarity between T1T4 and T1T0 indeed hindered Mandarin infants' discrimination, the more salient acoustic difference would be expected to allow Mandarin infants to discriminate T1T2 and T1T0.

\section{EXPERIMENT 3: MANDARIN INFANTS' DISCRIMINATION OF T1T2 AND T1TO}

\section{Stimuli}

The pseudoword /pansan/ was also used as stimuli. Infants were tested on their discrimination of T1T2 and T1T0. T1T2 and T1T0 carried saliently different pitch contours: T1T2 was "highlevel + mid-rising," but T1T0 was "high-level + mid-falling." As T0 was realized in a shortened duration, T1T0 exhibited a "long-short" duration pattern.

The same female Mandarin native speaker produced /pan1san2/ in IDS 20 times. Recordings were completed in the soundproof room of the phonetics lab at CASS, using Cool Edit Pro 2.0 at a sample rate of $16,000 \mathrm{~Hz}$.

Another five native Mandarin speakers judged the naturalness of the recordings on a continuum from 1 (extremely unnatural) to 5 (very natural). Two phoneticians selected the six most natural tokens of /pan $1 \operatorname{san} 2 /$. The six tokens of neutral tone (/pan $1 \operatorname{san} 0 /)$, which were used in Experiment 2, were also used in the present experiment. In the six tokens of each category, three were used in the habituation phase, and another three were used in the test phase.

For the canonical tone sequence of T1T2 (/pan1san2/), the average duration of the first syllable was $253.5 \mathrm{~ms}(S D=12)$, and the second syllable was $411.3 \mathrm{~ms}(S D=15.7)$. For the neutral tone sequence of T1T0 (/pan $1 \operatorname{san} 0 /)$, the average duration of the first syllable was $269.2 \mathrm{~ms}(S D=11.8)$, and the second syllable was $216 \mathrm{~ms}(S D=41.8)$. For tonal contours, 10 F0 values were 

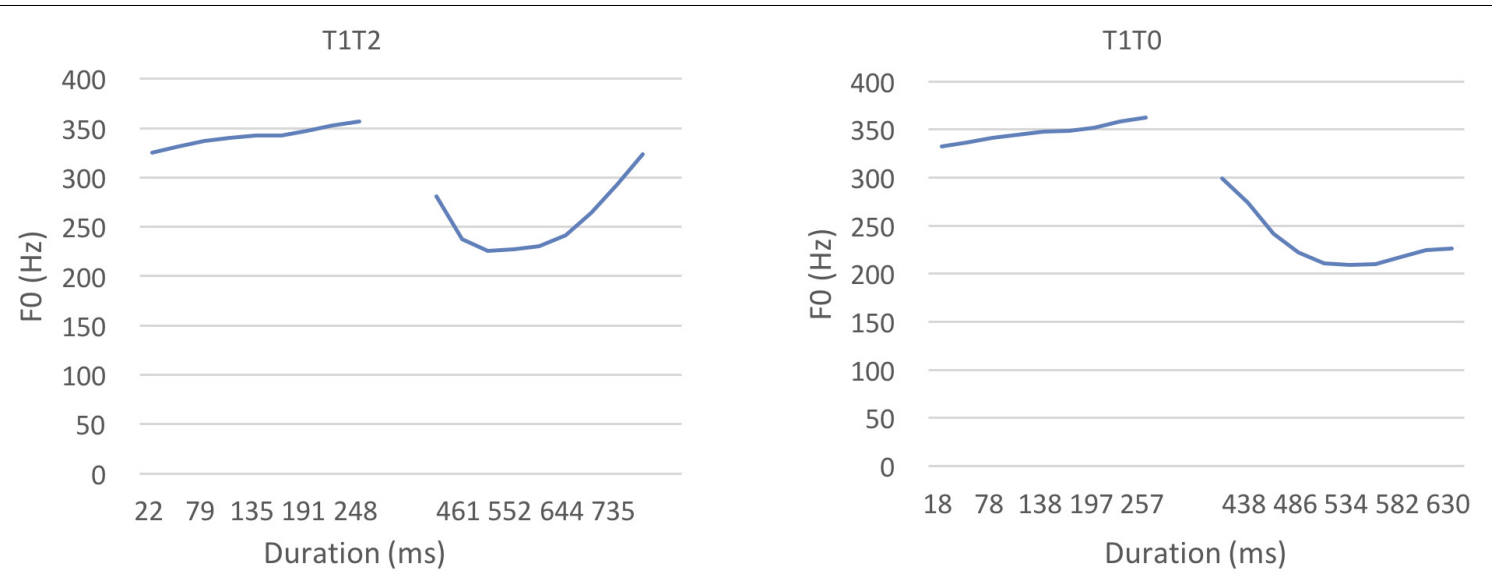

FIGURE 9 | Average FO contours of T1T2 and T1TO.

extracted on the F0 contour of each tone using Praat (Boersma and Weenink, 2013). For the T1T2 sequences, the maximal F0 value of T2 was $323.7 \mathrm{~Hz}$, and its minimal F0 value was $226 \mathrm{~Hz}$ with a range of $97.7 \mathrm{~Hz}$. For T1T0 sequences, as used in Experiment 2, the maximal of T0 was $303.9 \mathrm{~Hz}$, and its minimal F0 value was $218.5 \mathrm{~Hz}$ with a range of $85.4 \mathrm{~Hz}$. The average F0 contours are presented in Figure 9.

\section{Participants}

Thirty-five Mandarin-learning infants different from those in Experiment 2 were tested. Eight of them were 4-6 months old (mean age $=5 ; 16, S D=0.7,4$ males and 4 females) and 27 were 10-12 months old (mean age $=10 ; 9, S D=2.8,16$ males and 11 females). Another 34 infants were tested but later excluded due to fussiness $(N=10)$, parental intervention $(N=7)$, dialect interference in the input $(N=6)$, not being habituated $(N=4)$, equipment failure $(N=4)$, and experimenter error $(N=3)$. All Mandarin-learning infants were born and raised in Mandarin-speaking families where Mandarin was the only language in use. All parents reported normal hearing of the infants. Mandarin-learning infants were tested in the infant lab at CASS, Beijing.

\section{Procedures}

The experimental procedures were the same as in Experiment 2 (see section "Procedures" under the section "Experiment 2: Infants' Discrimination of Canonical Tone and Neutral Tone"). All experiments were completed in the infant lab at CASS, Beijing.

\section{Results}

To correct for skewness, the raw looking time was logarithmically transformed. Mandarin-learning infants were divided into two age groups: 4-6 months old and 10-12 months old. For each age group, the LogLT in the same trials and novel trials were compared.

We conducted the same analysis as in Experiment 2: a 2 (trial type: same/novel) $\times 2$ (habituated category: neutral tone/canonical tone) $\times 2$ (age group: 4-6/10-12 months old) mixed effect ANOVA was conducted. Trial type served as the within-subject factor. The between-subject factors were habituated category and age group. Trial type showed no main effect $[F(1,31)=1.68, p>0.05]$, nor did habituated category $[F(1,31)=0.03, p>0.05]$ or age group $[F(1,31)=0.42, p>0.05]$. There was also no interaction between trial type and age group, $F(1,31)=1.18, p>0.05$. The interaction between trial type and habituated category, however, was significant, $F(1,31)=4.53$, $p<0.05, \eta_{\mathrm{p}}^{2}=0.13$. No significant interaction was found among trial type, habituated category, and age group, $F(1,31)=0.22$, $p>0.05$.

We further split the data according to the habituated category to examine the interaction between trial type and habituated category. Paired $t$-tests were conducted to compare infants' looking time in the same trials and novel trials. When infants were habituated to canonical tones (T1T2, /pan1san2/), there was no difference between looking time in the same trials and novel trials $[t(14)=1.5, p>0.05]$. However, when infants were habituated to neutral tone (T1T0, /pan1san0/), their looking time in the novel trials was significantly longer than in the same trials $[t(19)=-2.51, p<0.05]$. Figure 10 shows the interaction between trial type and habituated category.

\section{Discussion}

When presented with an acoustically salient contrast (T1T2T1T0), neither the 4- to 6-month-old infants nor the 10- to 12-month-old infants showed a discrimination effect. Despite the fact that neutral and canonical tones are contrastive phonetically and phonologically, Mandarin-learning infants did not seem to discriminate the neutral-canonical tone contrast during their 1st year of life.

Nevertheless, when discriminating the T1T2 and T1T0 contrast, perceptual asymmetry was evident. When infants were habituated to the sequence of T1T0 (/pan1san0/), Mandarin infants discriminated neutral (T1T0, /pan1san0/) and canonical tones (T1T2, /pan1san2/) successfully. But when they were 


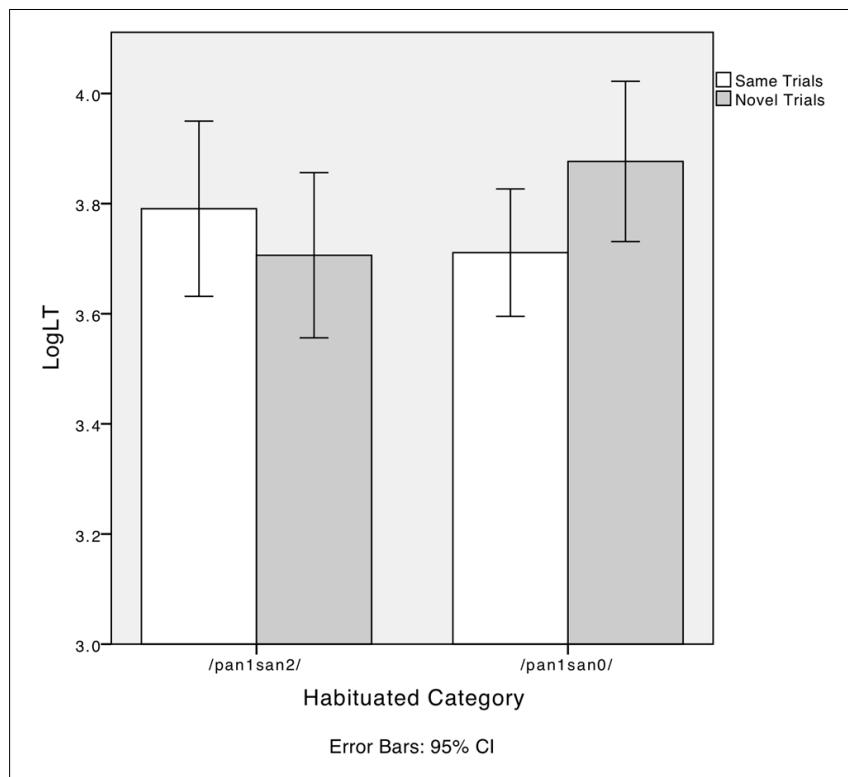

FIGURE 10 | Mandarin infants' log-transformed looking time (LogLT) in "same trials" and "novel trials" when habituated to canonical tone (/pan1san2/) or neutral tone (/pan1san0/).

habituated to the sequence of T1T2 (/pan1san2/), they did not show discrimination. The directional asymmetry might be related to the statistical distribution of tonal patterns in Mandarin, where canonical tones are used to distinguish lexical meanings and are therefore more common than neutral tones. About $3.8 \%$ of Mandarin vocabulary involves neutral tones (Zhu, 2009). Thus, habituating infants to the uncommon T1T0 may allow them to develop a representation of neutral tone, which would allow for later discrimination between T1T0 and T1T2. However, once habituated to the common T1T2, the uncommon T1T0 might be processed as a realization of $\mathrm{T} 1 \mathrm{~T} 2$.

\section{GENERAL DISCUSSION}

In the present study, we investigated the perception of neutral tone for tone- and non-tone (stress)- language listeners, both adults and infants. In Experiment 1, a discrimination task and an identification task were conducted for Mandarin and Dutch adult listeners. The results showed that Dutch adult listeners were able to discriminate between Mandarin neutral tones and canonical tones. In addition, Mandarin and Dutch listeners both identified neutral tones as unstressed. When presented with disyllabic sequences ending in a canonical tone, Mandarin listeners tended to identify the two syllables in the sequences as having "equal" stress, consistent with the claim that Mandarin does not have word level stress except for neutral tones.

In Experiments 2 and 3, we tested infants discrimination between neutral tone and canonical tone contrast using the visual fixation paradigm. In Experiment 2, Mandarin and Dutch infants were tested on the discrimination of a non-salient neutralcanonical tone contrast, namely T1T4 and T1T0. Results showed that Dutch infants could discriminate between neutral tones and canonical tones regardless of age. Because Dutch infants discriminated the contrast continuously, they might discriminate neutral-canonical contrast as lexical stress contrast, which exists in their native language. Perceptual asymmetry was found for Dutch infants: when they were habituated to the T1T0 sequence (/pan $1 \operatorname{san} 0 /)$, they discriminated canonical-neutral contrast; when they were habituated to the T1T4 sequence, however, they could not discriminate. Trochee is the predominant stress pattern in Dutch (van Heuven and Hagman, 1988; Leyden and van Heuven, 1996). For Dutch infants, the trochaic pattern (T1T0) may be more salient than the less frequent iambic pattern (T1T4). When presented with T1T0 first, it might be easier for the infants to consolidate the representation of the salient pattern, which allows for successful discrimination later. When presented with the iambic pattern, infants may accept the pattern as a non-prototypical realization of the trochaic pattern. Unexpectedly, however, neither the 4- to 6-month-old nor the 10- to 12-month-old Mandarin infants discriminated T1T4-T1T0 contrast. In previous studies, tonal discrimination was related to acoustic salience (Tsao, 2008; Liu and Kager, 2014; Chen and Kager, 2016). Both T4 (high-falling tone) and T0 (mid-falling tone) exhibited a falling tonal contour with a similar register. Even though Mandarin infants could form a prototype of falling tone through intensive habituation, they may perceive $\mathrm{T} 4$ and $\mathrm{T} 0$ as two realizations of the same tonal category.

To this end, a more salient contrast was used as stimuli in Experiment 3: T1T2 (/pan1san2/) and T1T0 (/pan1san0/). Compared with $\mathrm{T} 4$, the difference between $\mathrm{T} 2$ and $\mathrm{T} 0$ was larger in tonal contour with contradicting pitch movement directions ( $\mathrm{Li}$, 2017). The T1T2 is "high-level + midrising," and the T1T0 is "high-level + mid-falling." Although no discrimination was found overall, we found perceptual asymmetry to be similar to the Dutch infants in Experiment 2: when infants were habituated to T1T0 (/pan1san0/), they discriminated neutral-canonical tone contrast successfully; when habituated to T1T2 (/pan1san2/), infants failed to discriminate the contrast. Taken together, acoustic salience appeared to affect infants' discrimination. Perceptual asymmetry emerged when discriminating salient contrast. Directional asymmetry might reflect the statistical distribution of the tonal pattern in Mandarin. Canonical tones are more common in Mandarin, and neutral tones are more restricted in distribution. When habituated to the uncommon T1T0, it is likely that infants could form a new representation for neutral tone, thereby facilitating discrimination of the neutral-canonical tone contrast and leading to better discrimination.

Infants' different responses and perceptual asymmetry in Experiments 2 and 3 may reflect properties of their native languages, such as the statistical distribution of tonal/stress pattern in the input. In Dutch, duration is the most reliable cue for lexical stress. Like native Dutch adults, Dutch infants may perceive the T1T0-T1T4 contrast as lexical stress, where T4 carries a longer duration than T0. In Mandarin, however, 
lexical meanings are distinguished by pitch variations. The failed discrimination between T1T0 and T1T4 might reflect the fact that both T0 (mid-falling tone) and T4 (high-falling tone) were perceived as realizations of the same falling tone. It seems that 10 - to 12 -month-old infants already weigh phonetic cues according to their distribution in the input (Jusczyk et al., 1993).

Based on results from the infant experiments, one could conclude that Mandarin infants in the 1st year of life cannot discriminate between neutral tone and canonical tone contrast, although in Mandarin, neutral tones contrast with canonical tones phonetically and phonologically. Acoustic salience may also affect infants' discrimination. Mandarin infants showed perceptual asymmetry when presented with a salient contrast but not when presented with a non-salient contrast.

Particular neutral tone types may also influence infants' discrimination. In the present study, the neutral tone in the lexeme type carried the pattern of "XY", where X and Y stand for two different syllables. Under this condition, infants could only rely on phonetic cues to discriminate neutral and canonical tones without access to any morphological information. But in other contexts in which neutral tones are used, such as in reduplication and affixation, infants could predict neutral tones by using morphological cues, including the pattern of "XX" or "X/tsi0/" in which the second syllable is uttered in a neutral tone. Thus, the lexical neutral tone in the form of "XY" (Y can be said in either a neutral tone or a canonical tone, leading to different lexical meanings) might be more difficult to identify than the reduplication and affixation types. In Zhu (2002), a study of the production of neutral tones, reduplication occurred as early as 14 months but remained unstable at 24 months. Affixation and the lexeme types of neutral tones emerged at 17 months and stabilized earlier than reduplication. In the current research, we approached the discrimination of neutral tone and canonical tone contrast based mainly on phonetic cues. Reduplication and affixation types will be explored in future studies. Conceivably, infants may be able to represent and distinguish neutral tones from canonical tones in contexts where morphological markers are present.

The present study had two limitations. First, the sample size of 4- to 6-month-old Mandarin infants was small. However, previous studies reported that the discrimination performance of 10- to 12-month-old infants was better than 4- to 6-month-old infants for native contrast (Narayan et al., 2010; Shi, 2010). In this study, 10- to 12-month-old Mandarin infants were unable to discriminate the neutral tone and canonical tone contrast. Hence, we hypothesized that 4- to 6-month-old Mandarin infants would not have discriminated canonical-neutral tone contrast even if the sample size had been larger. Nevertheless, increasing the sample size would likely boost the statistical power of the results and produce an interaction between age group, trial type, and/or habituation category, rendering the developmental pattern more observable. Further testing of 4to 6-month-olds will be conducted when practicalities allow. We invite future studies for replication, and we leave the issue open for further investigation. Second, a potential factor affecting Mandarin and Dutch infants' perceptions is tonal variability. We used multiple tokens in the habituation for Mandarin and Dutch infants, expecting that Mandarin infants would be able to represent lexical tones phonologically rather than phonetically; however, our results did not support such a hypothesis. Dutch infants, on the other hand, were able to map variable tokens in the habituation to a single token in the test phase, suggesting to some extent that they had access to abstract representation of the disyllabic sequences. The failure of the Mandarin infants might be due to the much lower frequency of neutral tones than canonical tones in the input, which could have led to the infants' bias of accepting the variable tokens of neutral tones as realizations of canonical tones. Future studies may use single tokens to test Mandarin infants and investigate whether they can identify the difference between canonical and neutral tones on a phonetic level.

Several issues remain crucial for future studies. First, lexical knowledge might play a role in learning neutral tones in the lexeme type, and stimuli in a particular morphological structure may help highlight the developmental changes in perceiving neutral tones. For neutral tones in the context of reduplication (e.g., 妈妈, /ma1ma0/, mother) and affixation (e.g., 桌子, /tşuoltsı0/, table), infants could utilize morphological cues to perceive neutral tones. For neutral tones in the lexeme type (the stimuli used in the current study) cues other than phonetic ones might be needed to perceive neutral tone. Lexical meanings may facilitate infants' discrimination of neutral and canonical tones, but the infants tested in the current study were so young they had not yet developed sufficient knowledge of word meaning. As infants grow older, they may become capable of using word meaning to establish the representation of neutral tones. Future studies should test whether elder infants are able to use lexical meaning to discriminate between sequences ending in neutral and canonical tones.

Second, more attention should be paid to the perception of disyllabic tonal sequences. Disyllabic words are the predominant prosodic unit in Mandarin and occur more frequently than monosyllabic words (Feng, 1997; Wang, 2008). Hence, it is possible that infants learn disyllabic words holistically instead of the concatenation of individual tones, especially based on the observation that individual lexical tones are influenced by preceding and following tones due to articulation ( $\mathrm{Xu}$, 1997). Previous studies only used monosyllabic tones as stimuli (Mattock and Burnham, 2006; Mattock et al., 2008; Tsao, 2008; Chen, 2013; Liu and Kager, 2014; Chen and Kager, 2016), which may not reflect the actual language learning process. Knowledge of early perceptions of disyllabic canonical tone sequences among Mandarin infants will shed light on whether the acquisition of sequences involving neutral tones differ from those involving canonical tones.

In summary, a more detailed picture of neutral tone perception in future studies will emerge from the perspectives of phonetics, phonology, and word learning. Subsequent studies will provide deeper insights into discovering the process of suprasegmental information in early perception. 


\section{ETHICS STATEMENT}

This study was carried out in accordance with the recommendations of the Institute of Linguistics (CASS, China) and the Utrecht Institute of Linguistics OTS (Netherlands) with written informed consent from all adult participants/infants' parents. All participants/infants' parents gave written informed consent in accordance with the Declaration of Helsinki. The protocol was approved for ethics by the Institute of Linguistics (CASS, China) and the Utrecht Institute of Linguistics OTS (Netherlands).

\section{AUTHOR CONTRIBUTIONS}

$\mathrm{AL}, \mathrm{AC}$, and SF conceived and designed the study, and reviewed and edited the manuscript. SF performed the experiments and wrote the paper. All authors approved the manuscript.

\section{REFERENCES}

Bao, H., and Lin, M. (2014). Introduction of Experimental Phonetics. Beijing: Peking University Press.

Best, C. T. (1994). "The emergence of native-language phonological influences in infants: a perceptual assimilation model," The Development of Speech Perception: The Transition from Speech Sounds to Spoken Words, Vol. 167, eds J. C. Goodman and H. C. Nusbaum (Cambridge, MA: The MIT Press), 233-277.

Boersma, P., and Weenink, D. (2013). Praat: Doing Phonetics by Computer [Computer Program]. Version 5.3. Available at: http://www.praat.org/

Butler, J., Cortés, S., Correia, S., Uysal, E., Vigário, M., and Frota, S. (2015). European Portuguese- Learning Infants' Early Perception of Lexical Stress. Chicago, IL: Chicago Linguistic Society.

Cao, J. (1986). A study on neutral tone in Standard Chinese. Appl. Acoust. 4, 1-6.

Cao, J. (2008). A study on Mandarin lexical stress. Rep. Phon. Res. 20-29.

Chao, Y. (1979). A Grammar of Spoken Chinese. Beijing: The Commercial Press.

Chen, A. (2013). Universal Biases in the Perception of Mandarin Tones: From Infancy to Adulthood. Utrecht: Netherlands Graduate School of Linguistics.

Chen, A., and Kager, R. (2016). Discrimination of lexical tones in the first year of life. Infant Child Dev. 25, 426-439. doi: 10.1002/icd.1944

Chen, Y., and Xu, Y. (2006). Production of weak elements in speech - Evidence from F0 patterns of neutral tone in standard Chinese. Phonetica 63, 47-75. doi: $10.1159 / 000091406$

Cutler, A., and van Donselaar, W. (2001). Voornaam is not (really) a homophone: lexical prosody and lexical access in Dutch. Lang. Speech 44, 171-195. doi: $10.1177 / 00238309010440020301$

Deng, D. (2010). Experimental Study of Chinese Prosodic Word. Beijing: Peking University Press.

Duanmu, S. (2007). The Phonology of Standard Chinese, 2nd Edn. New York, NY: Oxford University Press.

Feng, S. L. (1997). Interactions between Morphology, Syntax and Prosody in Chinese. Beijing: Peking University Press.

Fikkert, P. (1993). "The acquisition of Dutch stress," in Amsterdam Series in Child Language Development, Vol. 1, eds M. Verrips and F. Wijnen (Amsterdam: University of Amsterdam Press).

Friederici, A. D., Friedrich, M., and Christophe, A. (2007). Brain responses in 4-month-old infants are already language specific. Curr. Biol. 17, 1208-1211. doi: 10.1016/j.cub.2007.06.011

Hohle, B., Bijeljac-Babic, R., Herold, B., Weissenborn, J., and Nazzi, T. (2009). Language specific prosodic preferences during the first half year of life: evidence from German and French infants. Infant Behav. Dev. 32, 262-274. doi: 10.1016/ j.infbeh.2009.03.004

Jia, Y. (2011). Stress pattern of disyllabic words terms with identical pronunciation and different morph-syntactic structures in Standard Chinese. J. Tsinghua Univ. 51, 1307-1312.

\section{FUNDING}

This study was supported by the National Natural Science Foundation of China (Nos. 61175016 and 61304250), the National Social Science Foundation of China (No. 15ZDB103), the CASS innovation project "Key Laboratory of Phonetics and Speech Science", and the China Exchange Program (CEP) between KNAW and CASS (No. 11CDP004).

\section{ACKNOWLEDGMENTS}

We thank Babylab group members at Utrecht University and Chinese Academy of Social Sciences for their help in designing the experiments, setting up the computer program, and recruiting participants. We sincerely thank all the families who participated in our research.

Jusczyk, P. W., Cutler, A., and Redanz, N. J. (1993). Infants' preference for the predominant stress patterns of English words. Child Dev. 46, 675-687. doi: $10.2307 / 1131210$

Keij, B., and Kager, R. (2013). "Early acquisition of word stress: a cross-linguistic infant study," in Proceedings of the International Child Psychology Conference, Nijmegen.

Kong, J., and Lü, S. (1998). "Study on tone patterns in Mandarin disyllabic words," in Proceedings of the 5th National Conference Man-Machine Speech Communication (NCMMSC, 1998), Harbin.

Lee, W.-S. (2003). "A phonetic study of the neutral tone in Beijing Mandarin," in Proceedings of the 15th International Congress of Phonetic Sciences (ICPHS 2003), Barcelona.

Leyden, K., and van Heuven, V. J. (1996). "Lexical stress and spoken word recognition: Dutch vs. English," in Linguistics in the Netherlands 1996, eds C. Cremers and M. den Dikken (Amsterdam: John Benjamins).

Li, A. (2017). Phonetic correlates of neutral tone in different information structures. Contemp. Linguist. 19, 348-378.

Li, A., and Fan, S. (2015). "Correlates of Chinese neutral tone perception in different contexts," in Proceedings of the 18th International Congress of Phonetic Sciences (ICPHS 2015), Glasgow.

Li, A., and Gao, J. (2017). "Production of neutral tone on disyllabic words by two-year-old Mandarin-speaking children," in Proceedings of the 11th International Seminar on Speech Production (ISSP 2017), Tianjin.

Lin, M. (2012). Experimental Study on Chinese Intonation. Beijing: China Social Science Press.

Lin, M., and Yan, J. (1980). The acoustic character of neutral tone in Beijing dialect. Dialect 3, 166-178.

Lin, T. (1983). Experiments on Properties of Neutral Tone in Linguistic Essays 1983. Beijing: The Commercial Press.

Lin, T., and Wang, L. (2013). A Course in Phonetics. Beijing: Peking University Press.

Liu, L., and Kager, R. (2014). Perception of tones by infants learning a non-tone language. Cognition 133, 385-394. doi: 10.1016/j.cognition.2014. 06.004

Lu, J., and Wang, J. (2005). On defining “Qingsheng (neutral tone)". Contemp. Linguist. 7, 107-112.

Luo, C., and Wang, J. (2002). An Outline of Common Phonetics. Beijing: The Commercial Press.

Mattock, K., and Burnham, D. (2006). Chinese and English infants' tone perception: evidence for perceptual reorganization. Infancy 10, 241-265. doi: 10.18632/oncotarget.22114

Mattock, K., Molnar, M., Polka, L., and Burnham, D. (2008). The developmental course of lexical tone perception in the first year of life. Cognition 106, 1367-1381. doi: 10.1016/j.cognition.2007.07.002 
Maye, J., Werker, J. F., and Gerken, L. (2002). Infant sensitivity to distributional information can affect phonetic discrimination. Cognition 82, B101-B111. doi: 10.1016/S0010-0277(01)00157-3

Narayan, C. R., Werker, J. K., and Beddor, P. S. (2010). The interaction between acoustic salience and language experience in developmental speech perception: evidence from nasal place discrimination. Dev. Sci. 13, 407-420. doi: 10.1111/j. 1467-7687.2009.00898.x

Polka, L., and Bohn, O. S. (1996). A cross-language comparison of vowel perception in English-learning and German-learning infants. J. Acoust. Soc. Am. 100, 577-592. doi: 10.1121/1.415884

Polka, L., and Bohn, O. S. (2003). Asymmetries in vowel perception. Speech Commun. 41, 221-231. doi: 10.1016/S0167-6393(02)00105-X

Polka, L., and Werker, J. F. (1994). Developmental changes in perception of nonnative vowel contrasts. J. Exp. Psychol. Hum. Percept. Perform. 20, 421-435. doi: 10.1037/0096-1523.20.2.421

Saffran, J. R., Aslin, R. N., and Newport, E. L. (1996). Statistical learning by 8-month-old infants. Science 274, 1926-1928. doi: 10.1126/science.274.5294. 1926

Sansavini, A., Bertoncini, J., and Giovanelli, G. (1997). Newborns discriminate the rhythm of multisyllabic stressed words. Dev. Psychol. 33, 3-11. doi: 10.1037/ 0012-1649.33.1.3

Segal, O., Houston, D., and Kishon-Rabin, L. (2016). Auditory discrimination of lexical stress patterns in hearing-impaired infants with cochlear implants compared with normal hearing: influence of acoustic cues and listening experience to the ambient language. Ear Hear. 37, 225-234. doi: 10.1097/AUD. 0000000000000243

Shi, R. (2010). Contextual variability and infants' perception of tonal categories. Chin. J. Phon. 2, 1-9.

Skoruppa, K., Pons, F., Bosch, L., Christophe, A., Cabrol, D., and Peperkamp, S. (2013). The development of word stress processing in French and Spanish infants. Lang. Learn. Dev. 9, 88-104. doi: 10.1080/15475441.2012.693881

Skoruppa, K., Pons, F., Christophe, A., Bosch, L., Dupoux, E., Sebastian-Galles, N., et al. (2009). Language-specific stress perception by 9-month-old French and Spanish infants. Dev. Sci. 12, 914-919. doi: 10.1111/j.1467-7687.2009. 00835.x

Sluijter, A. M., and van Heuven, V. J. (1995). Effects of focus distribution, pitch accent and lexical stress on the temporal organization of syllables in Dutch. Phonetica 52, 71-89. doi: 10.1159/000262061

Sluijter, A. M., and van Heuven, V. J. (1996). Spectral balances as an acoustic correlate of linguistic stress. J. Acoust. Soc. Am. 100, 2471-2485. doi: 10.1121/1. 417955

Sundara, M., Polka, L., and Genesee, F. (2006). Language-experience facilitates discrimination of /d-th/ in monolingual and bilingual acquisition of English. Cognition 100, 369-388. doi: 10.1016/j.cognition.2005.04.007

Tsao, F. (2008). The effect of acoustical similarity on lexical-tone perception of one-year-old Mandarin-learning infants. Chin. J. Psychol. 50, 111-124.

van Heuven, V. J., and de Jonge, M. (2011). Spectral and temporal reduction as stress cues in Dutch. Phonetica 68, 120-132. doi: 10.1159/000329900 van Heuven, V. J., and Hagman, P. (1988). "Lexical statistics and spoken word recognition in Dutch," in Linguistics in the Netherlands, eds P. Coopmans and A. Hulk (Dordrecht: Foris), 59-68.

van Ooijen, B., Bertoncini, J., Sansavini, A., and Mehler, J. (1997). Do weak syllables count for newborns? J. Acoust. Soc. Am. 102, 3735-3741. doi: 10.1121/1.420136

Veenker, T. J. G. (2013). The Zep Experiment Control Application (1.10) [Computer Software]. Beexy Behavioral Experiment Software. Available at: http://www. beexy.org/zep/

Wang, H. J. (2008). Non-linear Phonology of Chinese. Beijing: Peking University Press.

Wang, Y. (2004). The effects of pitch and duration on the perception of the neutral tone in standard Chinese. Acta Acust. 29, 453-461.

Weber, C., Hahne, A., Friedrich, M., and Friederici, A. D. (2004). Discrimination of word stress in early infant perception: electrophysiological evidence. Cogn. Brain Res. 18, 149-161. doi: 10.1016/j.cogbrainres.2003.10.001

Weber, C., Hahne, A., Friedrich, M., and Friederici, A. D. (2005). Reduced stress pattern discrimination in 5-month-olds as a marker of risk for later language impairment: neurophysiologial evidence. Cogn. Brain Res. 25, 180-187. doi: 10.1016/j.cogbrainres.2005.05.007

Wei, G. (2005). Neutral tone and weak stress in Beijing Mandarin and the transcription of the Chinese phonetic alphabet of putonghua. Stud. Chin. Lang. 6, 525-536+576.

Wu, Z. (1992). An Introduction to Phonetics in Standard Chinese. Beijing: Sinolingua.

Xu, Y. (1997). Contextual tonal variations in Mandarin. J. Phon. 25, 61-83. doi: 10.1006/jpho.1996.0034

Yang, S. (1989). The synthesis rules on Mandarin neutral tone. Appl. Acoust. 10, $12-18$.

Zhang, L., and Li, A. (2016). "Acoustic features of Mandarin neutral tone in five contexts," in Proceedings of the Fifth International Symposium on Tonal Aspects of Languages (TAL 2016), Buffalo, NY.

Zhong, X., Wang, B., Yang, Y., and Lü, S. (2001). The perception of stress in prosodic words of standard Chinese. Acta Psychol. Sin. 6, 481-488.

Zhu, H. (2002). Phonological Development in Specific Contexts: Studies of ChineseSpeaking Children. Clevedon: Multilingual Matters Ltd.

Zhu, H. Y. (2009). Neutral tone's character and its regulative principles. Appl. Linguist. 2, 34-41.

Conflict of Interest Statement: The authors declare that the research was conducted in the absence of any commercial or financial relationships that could be construed as a potential conflict of interest.

Copyright (C) 2018 Fan, Li and Chen. This is an open-access article distributed under the terms of the Creative Commons Attribution License (CC BY). The use, distribution or reproduction in other forums is permitted, provided the original author(s) and the copyright owner are credited and that the original publication in this journal is cited, in accordance with accepted academic practice. No use, distribution or reproduction is permitted which does not comply with these terms. 